\title{
A role for mucosal IL-22 production and Th22 cells in HIV-associated mucosal immunopathogenesis
}

\author{
CJ Kim ${ }^{1}$, A Nazli ${ }^{2}$, OL Rojas ${ }^{1}$, D Chege ${ }^{1}$, Z Alidina ${ }^{2}$, S Huibner ${ }^{1}$, S Mujib $^{1}$, E Benko ${ }^{3}$, C Kovacs ${ }^{2,3}$, \\ LYY Shin ${ }^{1}$, A Grin ${ }^{4}$, G Kandel ${ }^{1,5}$, M Loutfy ${ }^{3,6,7}$, M Ostrowski ${ }^{1,5,6}$, JL Gommerman ${ }^{1}$, C Kaushic ${ }^{2,3}$ \\ and $\mathrm{R} \mathrm{Kaul}{ }^{1,6,8}$
}

Interleukin-22 (IL-22) is a cytokine with epithelial reparative and regenerative properties that is produced by Th22 cells and by other immune cell subsets. Therefore, we explored the hypothesis that disruption of the gut barrier during HIV infection involves dysregulation of these cells in the gastrointestinal mucosa. Sigmoid IL-22-producing T cell and Th22 cells were dramatically depleted during chronic HIV infection, epithelial integrity was compromised, and microbial translocation was increased. These alterations were reversed after long-term antiretroviral therapy. While all mucosal IL-22-producing T-cell subsets were also depleted very early during HIV infection, at these early stages IL-22 production by non-T-cell populations (including NKp44 + cells) was increased and gut epithelial integrity was maintained. Circulating Th22 cells expressed a higher level of the HIV co-receptor/binding molecules CCR5 and $\alpha 4 \beta 7$ than CD4+ T-cell subsets in HIV-uninfected participants, but this was not the case after HIV infection. Finally, recombinant IL-22 was protective against HIV and tumor necrosis factor- $\alpha$-induced gut epithelial damage in a validated in vitro gut epithelial system. We conclude that reduced IL-22 production and Th22 depletion in the gut mucosa are important factors in HIV mucosal immunopathogenesis.

\section{INTRODUCTION}

Progressive HIV infection is characterized by a rapid and profound depletion of CD4 $\mathrm{T}$ cells from the gastrointestinal mucosa, as well as by structural deterioration of the gut epithelium and microbial translocation. ${ }^{1-5}$ Together, these factors may drive HIV disease progression. Despite the tremendous survival benefit that is conferred by effective antiretroviral therapy (ART), microbial translocation and systemic immune activation may remain elevated in ART treated individuals, and contribute to an elevated risk of death owing to cardiac disease and neurocognitive dysfunction. ${ }^{6-10}$

Gastrointestinal CD4 T-cell depletion may be a necessary component of HIV mucosal immunopathogenesis, but this alone is insufficient to cause clinical immunodeficiency, as demonstrated by nonhuman primate models. ${ }^{11,12}$ However, during pathogenic HIV infection this mucosal CD4 T-cell depletion is also accompanied by impaired epithelial barrier function and increased levels of microbial translocation. ${ }^{13,14}$ Recent literature indicates that the combination of structural epithelial deterioration and mucosal immunodeficiency are critical in driving HIV disease progression, ${ }^{15-18}$ yet little is known about how the epithelial barrier breaks down and why this leads to microbial translocation.

Nazli et al. demonstrated in vitro that HIV gp120 induces an innate tumor necrosis factor- $\alpha$ (TNF- $\alpha$ ) response by enterocytes, with subsequent breakdown of mucosal tight junction proteins. ${ }^{15}$ In pathogenic simian immunodeficiency virus infection, loss of colonic epithelial integrity was associated with increased lipopolysaccharide (LPS) levels in the lamina propria and draining lymph nodes, leading to bacteremia. ${ }^{17}$ Again, however, epithelial damage alone is insufficient to cause systemic microbial translocation, and plasma LPS levels were only elevated in humanized mice when epithelial breakdown was accompanied by simultaneous mucosal immunodeficiency. ${ }^{16}$ HIV is associated with mucosal depletion of specific T-cell subsets, such as microbe-responsive Th17 cells, as well as with impaired neutrophil recruitment and phagocytic macrophage

\footnotetext{
${ }^{1}$ Department of Medicine, University of Toronto, Toronto, Ontario, Canada. ²Department of Pathology and Molecular Medicine, McMaster University, Hamilton, Ontario, Canada. ${ }^{3}$ Maple Leaf Medical Clinic, Toronto, Ontario, Canada. ${ }^{4}$ Division of Gastroenterology, Department of Laboratory Medicine and Pathobiology, University of Toronto, Toronto, Ontario, Canada. ${ }^{5}$ St Michael's Hospital, Toronto, Ontario, Canada. ${ }^{6}$ Department of Immunology, University of Toronto, Toronto, Ontario, Canada. ${ }^{7}$ Women's College Hospital, Toronto, Ontario, Canada. ${ }^{8}$ Department of Medicine, University Health Network, Toronto, Ontario, Canada. Correspondence: CJ Kim (kim.connie@ gmail.com) or R Kaul (rupert.kaul@utoronto.ca)

Received 20 April 2012; accepted 22 June 2012; published online 1 August 2012. doi: 10.1038/mi.2012.72
} 
function, and this mucosal immune compromise may facilitate bacterial persistence in the gut mucosa and subsequent endotoxemia. ${ }^{16,18-20}$

Interleukin-22 (IL-22) is a member of the IL-10 cytokine family that is produced by $\mathrm{T}$ cells, monocytes, dendritic cells, natural killer (NK) cells, and innate lymphoid cells. ${ }^{21}$ IL-22 binds to a heterodimer consisting of IL-10R $\beta$ chain, signals through STAT3, and IL-22R is expressed predominantly on nonimmune cells of the skin, gut, and other organs. ${ }^{22-24}$ At mucosal surfaces, IL-22 provides innate immune protection against bacterial and fungal infections, as well as promoting inflammation and enhancing epithelial proliferation and repair. ${ }^{21,24,25}$ In experimental colitis, IL-22 regulated immune homeostasis and promoted mucosal wound healing, ${ }^{22,23}$ and in murine Citrobacter rodentium infection IL-22 regulated and maintained colonic lymphoid structure. ${ }^{26}$ While Th1 and Th17 cells commonly produce IL-22, recent studies have defined a Th22 CD4 T-cell subset that produces IL-22 independent of IFN- $\gamma$ and IL-17. ${ }^{27-29}$ These tissue reparative Th22 cells express IL-22 under the regulation of the aryl hydrocarbon receptor, and may share common surface markers with Th17 cells, such as the chemokine receptors CCR6 and CCR4, dipeptidylpeptidase IV CD26, and GPI-anchored protein CD90.27,29-32 Th22 cells have also been shown to express CCR 10 , potentially distinguishing them from the Th17 subset. ${ }^{27,29,30}$

We hypothesized that mucosal IL-22-producing cells and Th22 cells have an important role in maintaining the gut mucosal barrier, and that their depletion during HIV infection contributes to epithelial damage and microbial translocation.

\section{RESULTS}

\section{Study participants for gut mucosal studies}

Paired blood and sigmoid colonic biopsies were collected from 48 men in the following participant groups: HIV-uninfected (HIV -; $n=8$ ), HIV-infected, therapy-naïve (HIV +; $n=24$ ), and HIVinfected on long-term effective ART (HIV + ART; $n=16$ ). The HIV-infected, therapy-naïve group included participants during both early infection ( $<7$ months infection, $N=12$ ) and chronic infection ( $>1$ year infection; $N=12$ ). Criteria for early HIV infection were either documented seroconversion $(N=6)$ or a known high-risk HIV sexual exposure followed by symptoms of seroconversion illness $(N=6)$. All participants were HIV seropositive by the time of sample collection. Clinical parameters of study participants are summarized in Supplementary Table 1 online. The ART-naive HIV-infected group had a median blood CD4 count of 390 cells per $\mu \mathrm{l}$ and a blood viral load (VL) of 27,451 RNA copies per milliliter. Among HIV-infected, ART-naïve participants the early infected group had a higher CD4 count than those during chronic infection $(P=0.026)$, but the blood VL was comparable. ART-treated participants had been on therapy for a median of 12.5 years, with an undetectable blood $\mathrm{VL}$ ( $<50$ copies per $\mathrm{ml}$ ) for no less than 6 years and a median CD4 count of 660 cells per $\mu$ l.

\section{T-cell immune parameters in the blood and sigmoid colon} Immune parameters were assayed in freshly isolated mononuclear cells from the blood and sigmoid mucosa (Table 1).
Table 1 Descriptive immununological parameters of blood and sigmoid colon of the study cohort

\begin{tabular}{|c|c|c|c|}
\hline & HIV - & HIV + & HIV + ART \\
\hline \% Blood CD4 & $\begin{array}{c}63 \\
(34-72)\end{array}$ & $\begin{array}{c}32 \\
(14-65)^{\star \star a}\end{array}$ & $\begin{array}{c}48 \\
(8-70)^{\star b},{ }^{* c}\end{array}$ \\
\hline \% Sigmoid CD4 & $\begin{array}{c}58 \\
(28-74)\end{array}$ & $\begin{array}{c}15 \\
(3-43)^{\star \star a}\end{array}$ & $\begin{array}{c}49 \\
(21-60)^{\star \star b}\end{array}$ \\
\hline $\begin{array}{l}\text { Abs sigmoid CD4 } \\
\left.\text { ( } 10^{6} \text { cells/g tissue }\right)\end{array}$ & $\begin{array}{c}1.7 \\
(0.4-6.0)\end{array}$ & $\begin{array}{c}0.5 \\
(0.1-2.8)^{\star a}\end{array}$ & $\begin{array}{c}0.9 \\
(0.1-2.8)\end{array}$ \\
\hline \% Blood CD8 & $\begin{array}{c}33 \\
(22-55)\end{array}$ & $\begin{array}{c}54 \\
(30-82)^{\star a}\end{array}$ & $\begin{array}{c}42 \\
(28-73)^{\star \mathrm{C}}\end{array}$ \\
\hline \% Sigmoid CD8 & $\begin{array}{c}27 \\
(17-45)\end{array}$ & $\begin{array}{c}67 \\
(37-90)^{\star \star a}\end{array}$ & $\begin{array}{c}36 \\
(10-53)^{\star * b}\end{array}$ \\
\hline $\begin{array}{l}\text { Abs sigmoid CD8 } \\
\left.\text { ( } 10^{6} \text { cells/g tissue }\right)\end{array}$ & $\begin{array}{c}0.9 \\
(0.3-1.6)\end{array}$ & $\begin{array}{c}2.0 \\
(0.8-9.1)^{\star * a}\end{array}$ & $\begin{array}{c}0.6 \\
(0.1-1.9)^{\star * b}\end{array}$ \\
\hline $\begin{array}{l}\text { \% Blood CCR5 + } \\
\text { CD4 T cells }\end{array}$ & $\begin{array}{c}1.5 \\
(0.1-7.0)\end{array}$ & $\begin{array}{c}4.1 \\
(0.1-15.3)\end{array}$ & $\begin{array}{c}1.5 \\
(0.1-8.3)^{\star \mathrm{b}}\end{array}$ \\
\hline $\begin{array}{l}\text { \% Sigmoid CCR5 + } \\
\text { CD4 T cells }\end{array}$ & $\begin{array}{c}42 \\
(36-67)\end{array}$ & $\begin{array}{c}22.5 \\
(6-71)^{\star a}\end{array}$ & $\begin{array}{c}42 \\
(17-70)^{\star b}\end{array}$ \\
\hline $\begin{array}{l}\text { \% Blood HLADR + } \\
\text { CD4 T cells }\end{array}$ & $\begin{array}{c}5.9 \\
(2.3-20)\end{array}$ & $\begin{array}{c}16 \\
(5-46)^{\star a}\end{array}$ & $\begin{array}{c}8.6 \\
(3.7-27)\end{array}$ \\
\hline $\begin{array}{l}\text { \% Sigmoid HLADR + } \\
\text { CD4 T cells }\end{array}$ & $\begin{array}{c}13 \\
(5-39)\end{array}$ & $\begin{array}{c}30 \\
(10-56)^{\star a}\end{array}$ & $\begin{array}{c}15 \\
(5-28)^{\star \star b}\end{array}$ \\
\hline $\begin{array}{l}\% \text { Blood HLADR + } \\
\text { CD8 T cells }\end{array}$ & $\begin{array}{c}26 \\
(11-36)\end{array}$ & $\begin{array}{c}69 \\
(11-86)^{\star a}\end{array}$ & $\begin{array}{c}14 \\
(5.4-46)^{\star \star b}\end{array}$ \\
\hline $\begin{array}{l}\text { \% Sigmoid HLADR + } \\
\text { CD8 T cells }\end{array}$ & $\begin{array}{c}33 \\
(8-60)\end{array}$ & $\begin{array}{c}75 \\
(15-92)^{\star \star a}\end{array}$ & $\begin{array}{c}17 \\
(2.3-52)^{\star * b}\end{array}$ \\
\hline
\end{tabular}

Abbreviation: Abs, absolute.

Values indicate median (range).

Significance of $P \leqslant 0.05$ is indicated by *, and $P \leqslant 0.001$ is indicated by **. aHIV - to HIV + .

bHIV + to HIV + ART

CHIV - to HIV + ART

Untreated HIV infection was associated with preferential CD4 + T-cell depletion and with an equivalent increase in immune activation (HLA-DR expression) in both the blood and gut mucosa. CCR5 + CD $4+$ T cells were depleted within the sigmoid mucosa $(P=0.004)$, but not in blood $(P=0.151)$. While early HIV-infected participants tended to have a higher \%CD4 in blood (early, $36.8 \%$ vs. chronic, $24.0 \% ; P=0.078)$ and less blood CD4 activation than the chronic infection group (11.1 vs. $18.9 \% ; P=0.027$ ), mucosal CD4 depletion was comparable ( 17.2 vs. $13.4 \% ; P=0.106$ ). CD4 reconstitution was incomplete in the blood despite long-term ART $(P=0.023)$, and also tended to be lower in the sigmoid mucosa (proportion, $P=0.081$; absolute number, $P=0.086$ ). Expression of the immune activation marker HLADR did not differ between ART-treated and HIV-uninfected participants.

\section{Preferential depletion of sigmoid Th22 cells during HIV infection and reconstitution on ART}

Sigmoid Th22 cells were defined as CD4 $+\mathrm{T}$ cells producing IL-22 in the absence of IL-17a or IFN- $\gamma$, and Th17 and Th1 cells were defined by their capacity to produce IL-17a and 

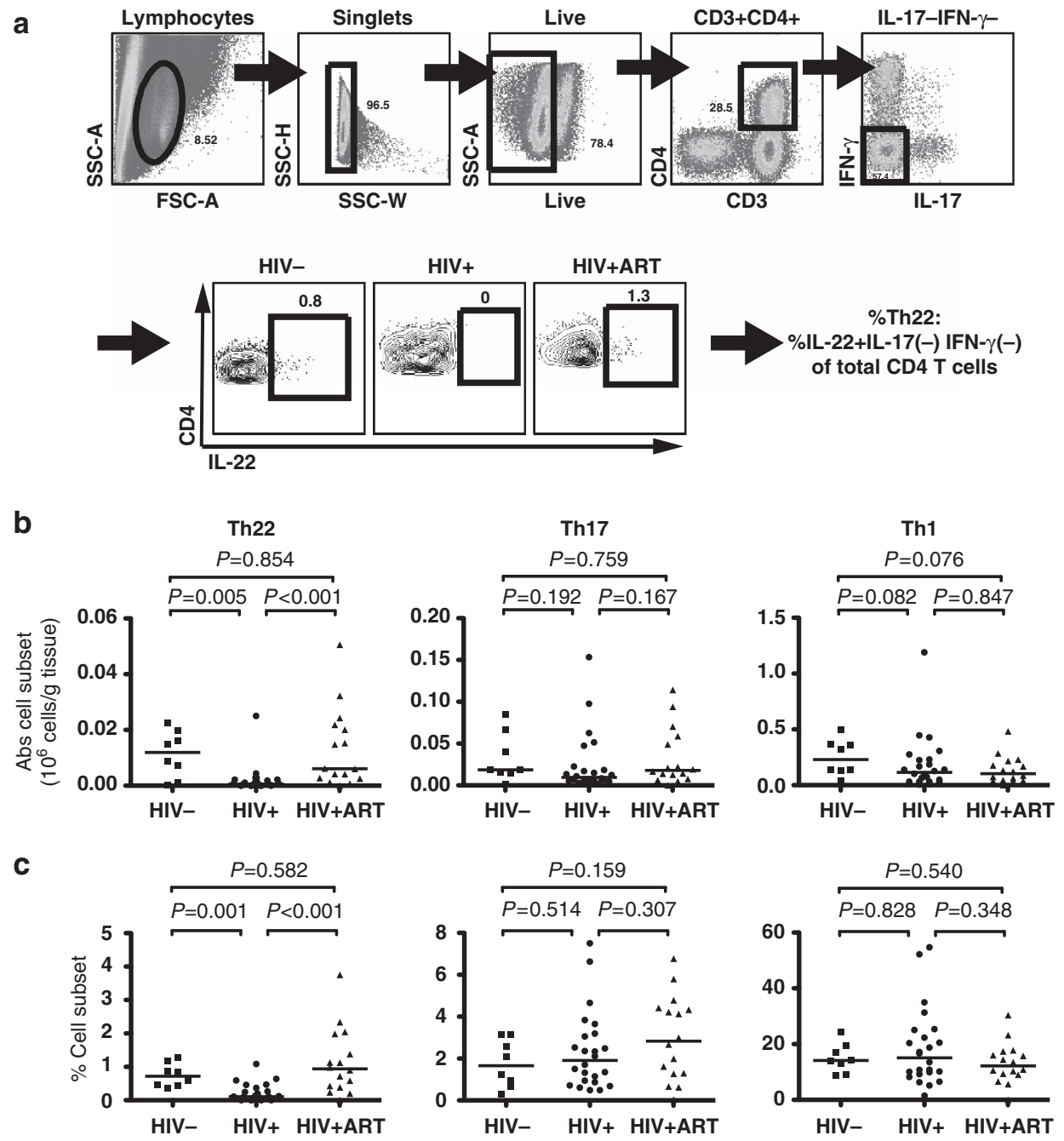

Figure 1 Th22 cells were preferentially depleted in the sigmoid colon of the HIV-infected group and reconstituted in the antiretroviral therapy (ART)-treated group. (a) Gating strategy of sigmoid Th22 cells and representative flow cytometry plots. (b) Absolute numbers (106 cells/g tissue) and (c) frequency (\%) of Th22 cells, Th17, and Th1 from the sigmoid colon.

IFN- $\gamma$, respectively (Figure 1a). In HIV-uninfected donors, Th22 cells were enriched eightfold in the sigmoid colon compared with blood $(0.72 \%$ vs. $0.09 \%$, respectively, $P=0.012)$ and the following sigmoid CD4 T-cell subsets were responsible for IL-22 production: Th1 cells (48.7\%), Th22 cells (35.5\%), and Th17 cells (27.3\%). Most Th22 cells displayed an effector memory phenotype (CD45RO + CCR7 - ) in both the sigmoid mucosa $(\sim 85 \%)$ and blood ( 70\%).

There was a substantial reduction in both the absolute number and frequency of Th22 cells in the sigmoid mucosa during untreated HIV infection compared with both HIV-uninfected ( $P=0.005$ and $P=0.001$, respectively) and ART-treated groups (both $P<0.001$; Figure $\mathbf{1 b}$ and $\mathbf{c}$ ). Although there was substantial interindividual heterogeneity in Th22 reconstitution after long-term ART, the degree of Th22 reconstitution was not associated with ART regimen or duration, or blood CD4 count (data not shown). The frequency and the absolute number of sigmoid Th1 cell subsets were comparable across the study groups. Unexpectedly, the mucosal Th17 frequency was comparable across study groups, although the Th17 cell number was depleted during chronic (not early) HIV infection (HIV - , $18.5 \times 10^{-3}$ cells per g tissue, chronic HIV,$+ 8.9 \times 10^{-3}$ cells per g tissue; $P=0.045$ ).

Immunohistochemical studies were performed on flashfrozen sigmoid biopsies to confirm Th22 depletion in HIV-infected participants. However, IL-22 expression was uncommon in the absence of phorbol 12-myristate 13-acetate and ionomycin stimulation. Staining for CCR10, a recently identified phenotypic marker of Th2 2 cells, ${ }^{30}$ we first confirmed that the rare mucosal CD3/IL-22 double-positive cells did co-express CCR10 (Supplementary Figure 1a online), and then used CD3/CCR10 co-expression as a proxy for Th22 cells. In HIVuninfected sigmoid biopsies, clusters of CD3/CCR10 co-positive cells were often observed; HIV-infected biopsies contained far fewer co-stained cells, although single-stained cells for each marker were present (Supplementary Figure 1b online). 
Overall, there was clear preferential depletion of CD3/CCR10 co-staining (Th22) cells in the sigmoid colon during HIV infection, above and beyond the generalized mucosal CD4 + depletion.

The frequency of sigmoid Th22 cells correlated with an increased overall gut $\mathrm{CD} 4$ cell percentage $(P=0.025$; Spearman correlation coefficient, $R_{\mathrm{S}}=0.456$ ). In addition, the absolute number of sigmoid Th22 cells was associated with higher blood CD 4 count $\left(P=0.045 ; R_{\mathrm{S}}=0.412\right)$, and higher blood and gut CD4 percentage (blood, $P=0.020 ; R_{\mathrm{S}}=0.471$; gut, $P=0.017$; $\left.R_{\mathrm{S}}=0.482\right)$. Neither the frequency nor the absolute number of mucosal Th22 cells was associated with blood VL.

In the blood compartment, frequency of Th1 and Th17 cells were comparable across the study groups, and Th22 cells were increased in ART-treated individuals compared with ARTnaïve individuals ( $P=0.014$; Supplementary Figure 2 online). Recent studies have identified CD26 and CD90 as surface markers that identify Th17 cells, including Th17 cells coproducing IL-22. ${ }^{31,32}$ However, while blood Th22 cells expressed high levels of CD26, similar to Th17 cells (Th22, 91.1\% vs. Th17, 77.9\%), CD90 expression was infrequent on Th22 cells (Th22, $0.92 \%$ vs. Th17, $6.56 \%$; data not shown). Plasma levels of IL22 were measured by a commercially available IL-22 ELISA kit (R\&D Systems), but IL-22 concentrations were below the limit of detection (range, 0-6 $\mathrm{pg} \mathrm{ml}^{-1}$ ) in both the HIV-uninfected and HIV-infected groups.

\section{IL-22 production by other mucosal cell subsets}

Although Th22 cells were preferentially depleted from the sigmoid mucosa during HIV infection, these are not the only mucosal source of IL-22. ${ }^{21}$ There was a dramatic reduction in the overall frequency of IL-22-producing gut lymphocytes, $\mathrm{CD} 3+$ cells, and CD4 $(-) \mathrm{T}$ cells during all stages of untreated HIV infection, and as well, IL-22-producing capacity from bulk $\mathrm{CD} 4+$, Th1 cells, and Th17 cells was reduced (Figure 2). IL-22 production by all mucosal immune subsets was reconstituted after long-term ART.

\section{IL-22 dysregulation during the early stages of HIV infection}

There was clear, preferential depletion of the mucosal Th22 subset during early HIV infection (Figure $\mathbf{3 a}$ and $\mathbf{b}$ ), and total CD3 + T-cell IL-22-producing capacity was also reduced during early HIV infection $(P=0.002$; Figure $3 c)$. In addition, IL-22 production was reduced within bulk CD4 + T cells (HIV,$- 2.1 \%$ vs. early HIV,$+ 0.7 \% ; P=0.017)$, bulk CD $4(-)$ cells ( 1.4 vs. $0.1 \% ; P=0.001$ ), Th1 (6.0 vs. $1.7 \% ; P=0.005$ ), and Th17 subsets ( 27.6 vs. $12.1 \% ; P=0.021)$. However, while we observed a dramatic reduction in IL-22 production capacity from all CD3 + T-cell subsets during early HIV infection, this HIV stage was uniquely characterized by the enhanced production of IL-22 from a sigmoid population of IL-22 + CD3(-) cells (Figure 3d and e). A subset of these cells was CD4+ (median, 12.5\%). In three ART-naïve HIV-infected participants, we assessed the NK cell markers CD56 and NKp44; while most IL-22 + CD3(-) cells were CD56(-), a substantial subset expressed NKp44+ (16.7, 45.0, and 77.8\%; data not shown).

\section{Sigmoid epithelial integrity at different stages of} HIV infection

We examined epithelial integrity in serially sectioned sigmoid biopsies by immunofluorescence analysis of tight junction proteins ZO-1 and claudin-2 (green), cytokeratin (red), and 46-diamidino-2-phenylindole (DAPI; blue). Epithelial damage was defined as ZO-1 reduction and increased pore-forming protein claudin-2 expression. Although heterogeneity was apparent in each group, biopsies from HIV-uninfected individuals showed continuous and robust expression of ZO-1 and minimal expression of claudin-2. Untreated chronic HIV infection was associated with multifocal disruption and reduced expression of ZO-1 and increased expression of claudin-2 in the epithelium. In contrast, epithelial integrity was maintained during early HIV infection. Three representative images of $\mathrm{ZO}-1$ and claudin-2 are shown for each cohort: $\mathrm{HIV}-$, early $\mathrm{HIV}+$, and chronic $\mathrm{HIV}+($ Figure 4).

\section{Plasma markers of microbial translocation during HIV infection}

Plasma LPS and soluble CD14 (sCD14) are markers of microbial translocation that are increased during HIV infection. ${ }^{13,33}$ Plasma LPS was increased only during chronic, untreated HIV infection $(P=0.025)$, but not in early HIV infection $(P=0.817)$ or in long-term ART-treated individuals ( $P=0.188$; Supplementary Figure 3a online). sCD14 was elevated in all HIV-infected groups, regardless of disease stage (early, $P=0.031$; chronic, $P=0.002)$ or treatment status $(P=0.003$; Supplementary Figure $\mathbf{3 b}$ online). Neither the frequency nor the absolute number of sigmoid Th22 cells were associated with plasma LPS or sCD14 levels.

\section{Circulating Th22 cells constitute a highly HIV-susceptible CD4+ subset}

Overall, there was dramatic depletion of Th22 cells and other IL-22 + cell subsets during untreated HIV infection. To investigate this further, levels of the HIV co-receptor CCR5 and the HIV-binding and gut-homing integrin $\alpha 4 \beta 7$ were compared between circulating bulk CD $4+$ memory $(\mathrm{CD} 45 \mathrm{RO}+)$, Th1, Th17, and Th22 cells from HIV-uninfected $(n=10)$ and ART-naive, chronically HIV-infected individuals $(n=6)$. In contrast to the sigmoid mucosa, most IL-22-producing CD4+ $\mathrm{T}$ cells in the blood of HIV-uninfected donors were true Th22 cells $(71.9 \%)$, with a lower proportion of IL-22 produced by Th1 (12.3\%) and Th17 cells (14.8\%).

In HIV-uninfected individuals, CCR5 frequency was higher in Th22 cells than bulk CD4 + or Th1 cells, while similar to Th17 cells, and $\beta 7^{\text {high }}$ expression by Th22 cells was more frequent than by bulk CD $4+\mathrm{T}$ cells but similar to Th1 cells and Th17 cells (Figure 5a and b; gating strategy and representative flow plots, Supplementary Figure 4 online). The mean fluorescence intensity of CCR 5 was at least 7.2-fold higher on Th22 cells than on any other subsets and the mean fluorescence intensity of $\beta 7$ was at least 1.8-fold higher than other subset (data not shown). While CCR 5 and $\beta 7^{\text {high }}$ expression were increased on all IL-22 + CD4 cells compared with bulk CD 4 cells (both $P=0.005$ ), Th22 cells 
a

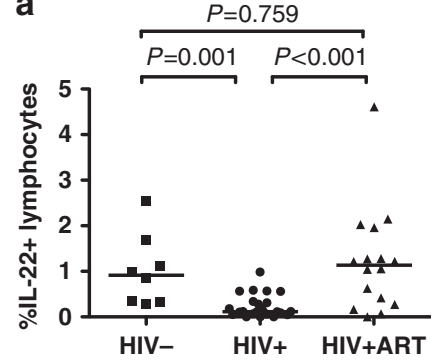

d

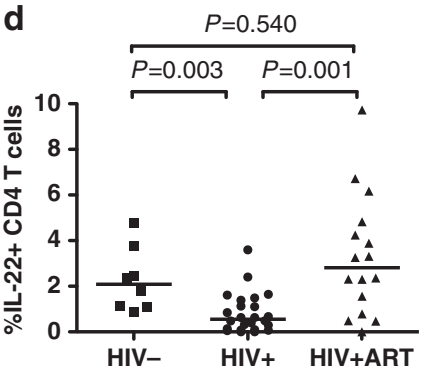

b

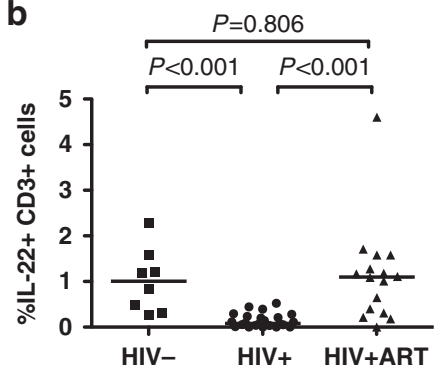

e
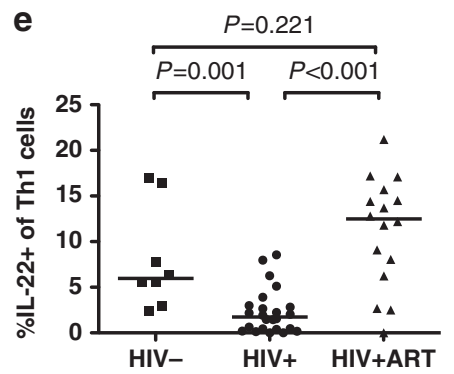

CD4 T cells
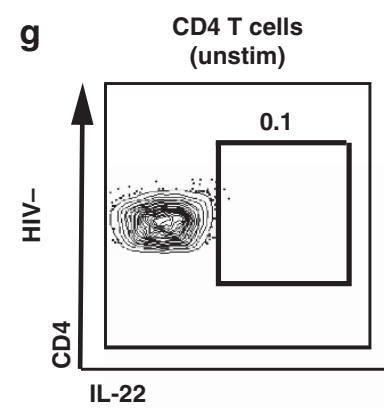

$\stackrel{+}{ \pm}$

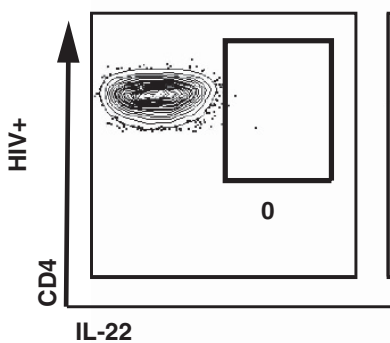

$\stackrel{+}{+}$

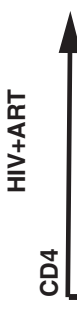

4

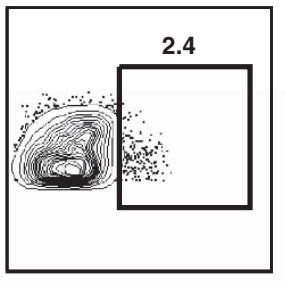

Th1 cells
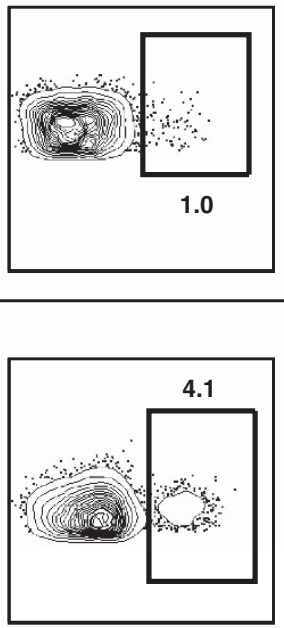
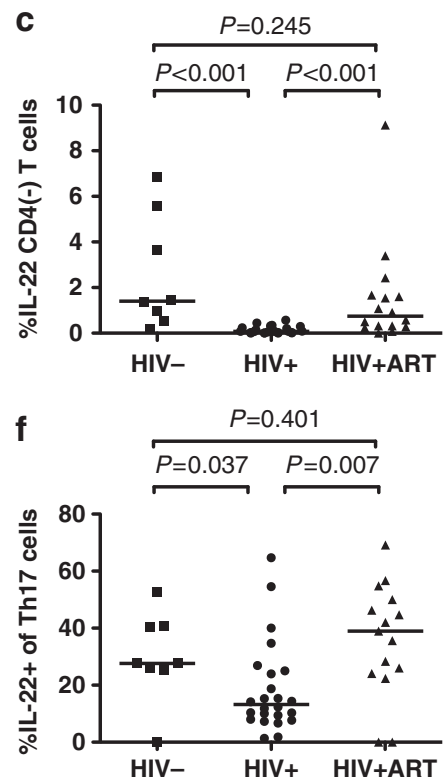

Th17 cells
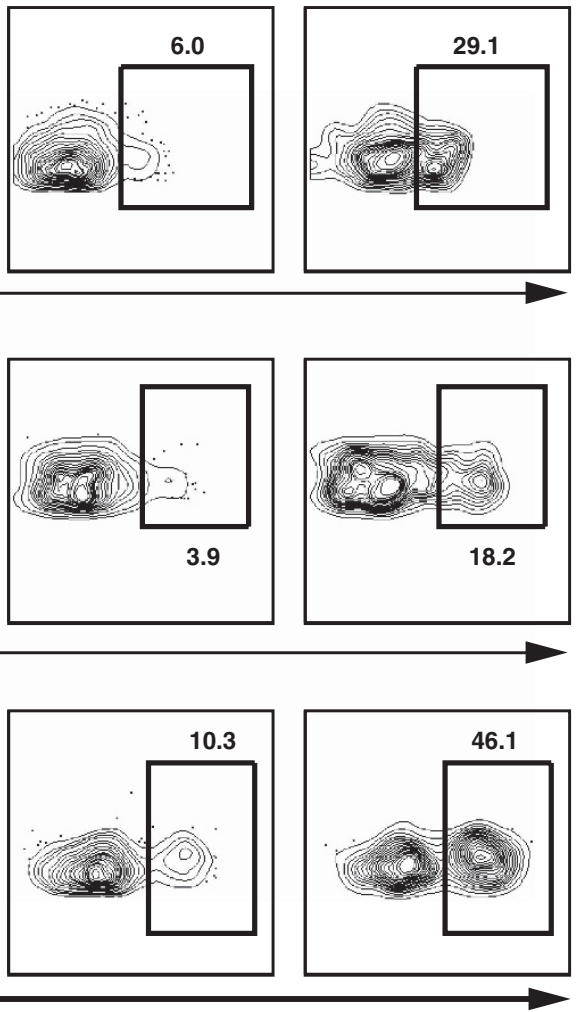

Figure 2 Mucosal IL-22 production by various lymphocyte subsets was lost. IL-22 from (a) gut lymphocytes, (b) CD3+ T cells, (c) CD4(-) T cells, (d) CD4+ T cells, (e) Th1 cells, and (f) Th17 cells was depleted in HIV-infected but not antiretroviral therapy (ART)-treated participants. (g) Flow cytometry plots including unstimulated controls for representative participant in each study group are shown. unstim, unstimulated.

had particularly high CCR5 and $\beta 7$ mean fluorescence intensity compared with total IL-22-producing CD4 cells $(P=0.009$ and $P=0.028$, respectively; data not shown). Interestingly, no increased expression of CCR 5 or $\beta 7^{\text {high }}$ expression was seen on blood Th2 2 cells after HIV infection (Figure $\mathbf{5 c}$ and $\mathbf{d}$ ), and there was a selective HIV-associated reduction in the expression of both CCR 5 and $\beta 7$ by Th 22 cells in the context of untreated HIV infection (Figure 5e and f). 

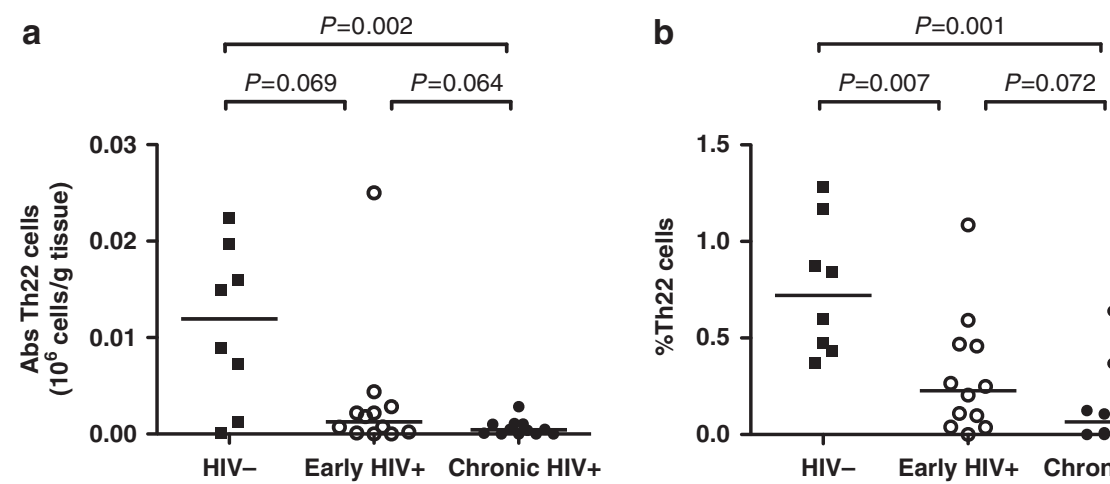

C
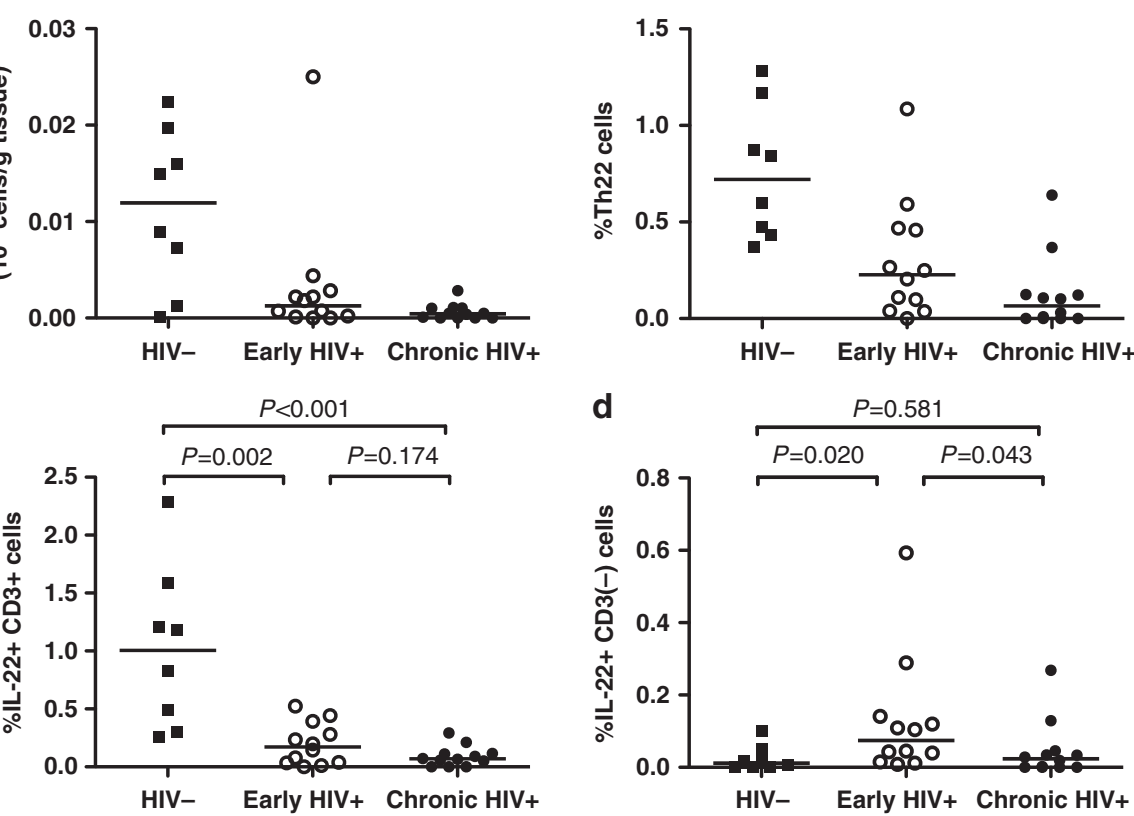

e

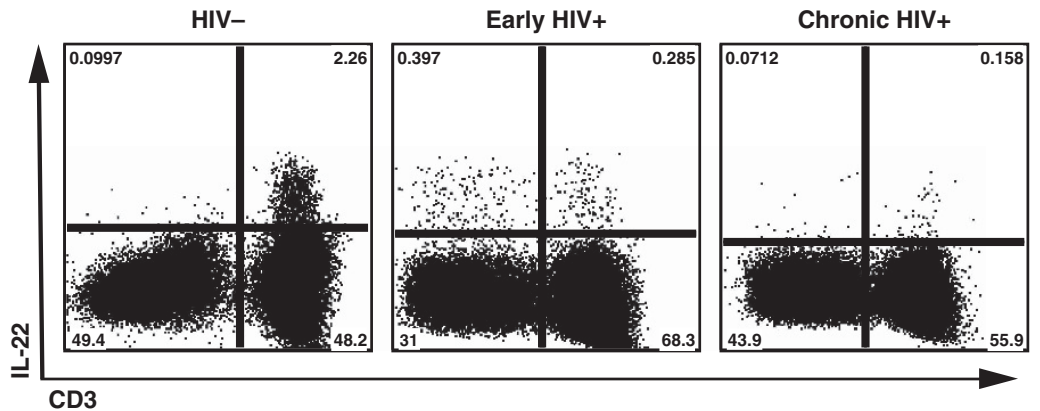

Figure 3 Early HIV-infected individuals had reduced sigmoid Th22 cells and IL-22 production capacity from CD3+ T cells, but increased IL-22 from CD3(-) subsets. (a) Absolute and (b) proportion of Th22 cells was depleted in early and chronic HIV-infected individuals, (c) as well as IL-22 depletion from bulk T cells ( $\mathbf{d}$ and $\mathbf{e}$ ). However, early HIV-infected individuals uniquely increased their capacity to produce IL-22 from CD3(-) lymphocytes.

\section{In vitro effects of IL-22 on HIV and TNF- $\alpha$-induced epithelial damage}

Based on these results, we examined the ability of recombinant IL-22 and/or IL-17a to prevent HIV and TNF- $\alpha$-mediated epithelial damage in an in vitro enterocyte system that has been previously validated. ${ }^{15}$ T84 intestinal monolayers were pretreated with IL-17a, IL-22, or both cytokines for $2 \mathrm{~h}$, and HIV (ADA strain) added, or in a separate experiment cytokines were cocultured with TNF- $\alpha$ for 24 and $48 \mathrm{~h}$, and transepithelial resistance (TER) was measured. By $24 \mathrm{~h}$, treatment with HIV or TNF- $\alpha$ alone had induced a profound reduction in epithelial integrity $\left(P<0.001\right.$; Figure 6) ${ }^{15}$ The TER reduction at 24 h was completely abrogated by IL-22 alone (both $P<0.001$ ), and was attenuated to a lesser degree by IL-17a (HIV, $P=0.051$; TNF- $\alpha, P<0.01$ ) or both cytokines combined (HIV, $P=0.001$; TNF- $\alpha, P<0.001$; Figure $6 \mathbf{a}$ and $\mathbf{b}$ ); at $48 \mathrm{~h}$, TER loss was effectively prevented by all three cytokine conditions (all
$P<0.001$, data not shown). These TER findings were confirmed through immunofluorescence staining of the tight junction protein ZO-1 (Figure 6c). Overall, both exogenous IL-17a and IL-22 protected against HIV and TNF- $\alpha$-induced epithelial barrier loss in this model system, with IL-22 demonstrating more rapid and complete protection.

\section{DISCUSSION}

HIV infection is associated with the depletion and/or dysfunction of key mucosal effector cells and with direct damage to the epithelial barrier. ${ }^{1,15,18}$ While poorly understood, the simultaneous and synergistic occurrence of both factors is likely required for the development of microbial translocation and the systemic immune activation. ${ }^{13}$ These pathogenic events in the gastrointestinal mucosa are thought to have a central role in HIV pathogenesis, and we now provide the first evidence that gut IL-22-producing and Th22 cells link these two aspects of 
a

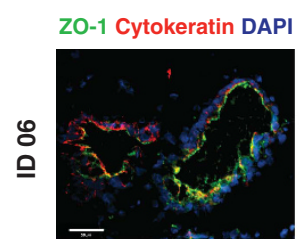

ช

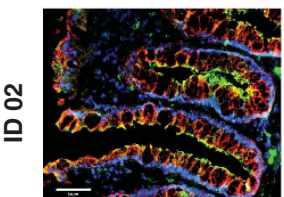

\&

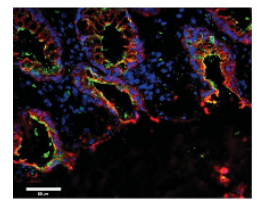

b
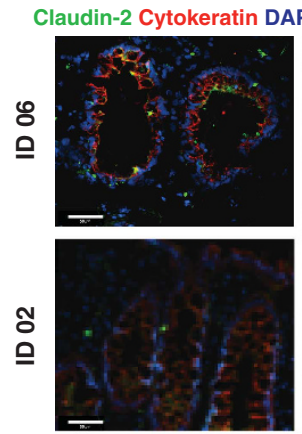

ஃ

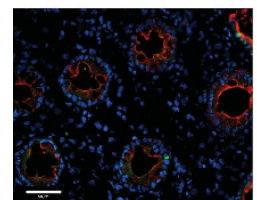

HIV-

ZO-1 DAPI
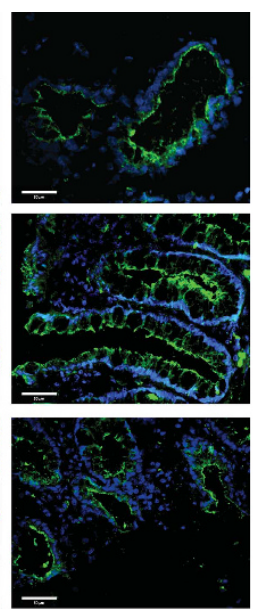

HIV-
Early HIV+
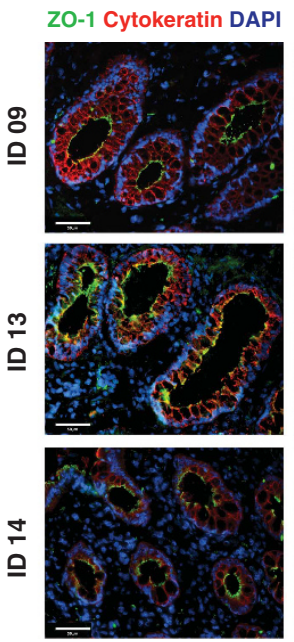

Early HIV+

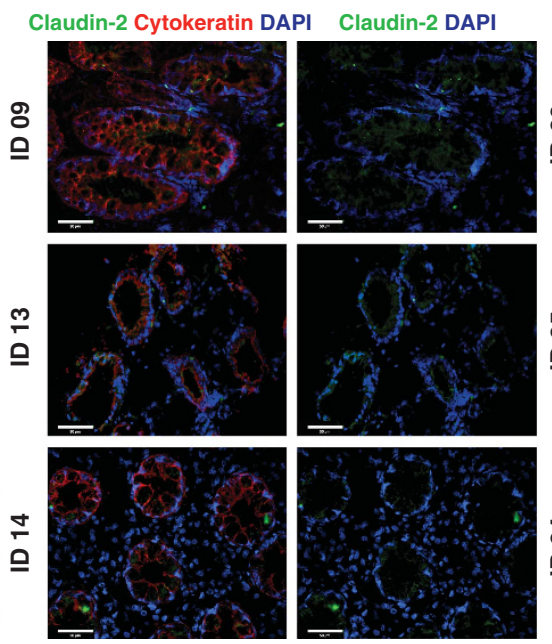

ZO-1 DAPI
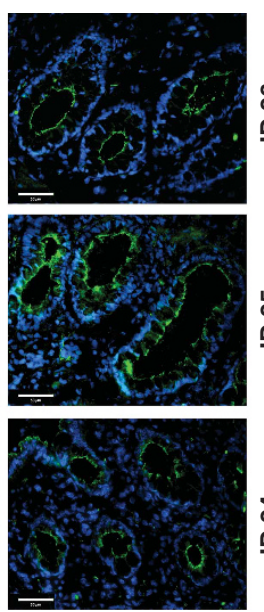
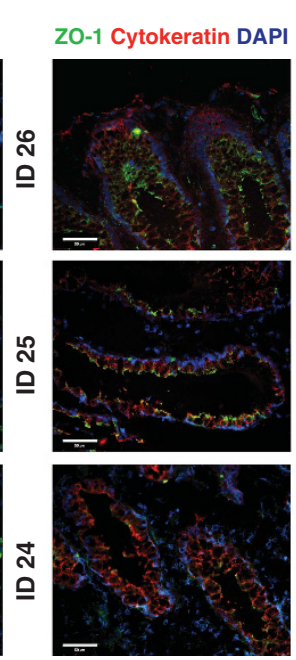

ZO-1 DAPI
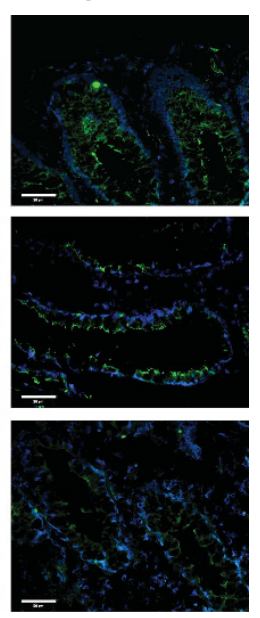

Chronic HIV+
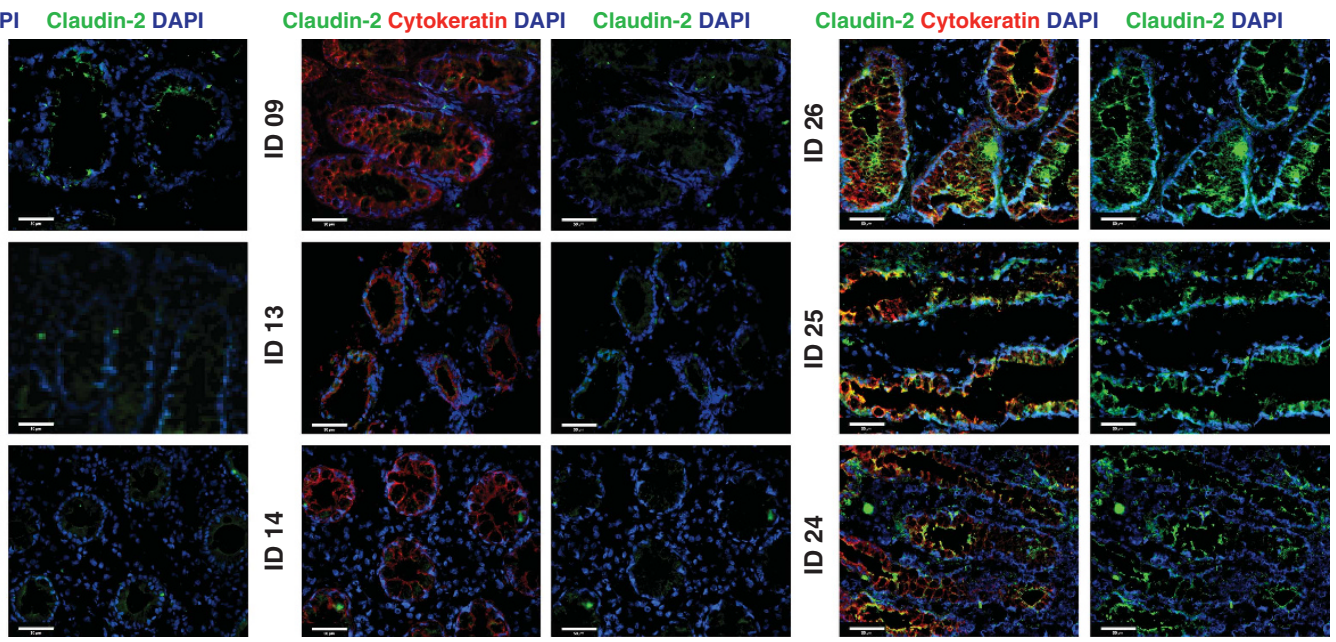

Figure 4 Immunofluorescence analysis of epithelial integrity of HIV-uninfected, and early and chronic HIV-infected participants. Sigmoid slides were stained with ZO-1 (green), claudin-2 (green), cytokeratin (red), and 46-diamidino-2-phenylindole (DAPI) (blue), and imaged at $\times 200$ original magnification. Representative images of three HIV-uninfected (ID 06, ID 02, and ID 08), three early HIV-infected (ID 09, ID 13, and ID 14), and three chronic HIV-infected (ID 26, ID 25, and ID 24) participants demonstrate (a) multifocal disruption and reduced expression of ZO-1 and (b) an increase in the pore-forming protein claudin-2 in chronic HIV infection, while early HIV-infected participants maintained gut integrity.

HIV mucosal immunopathogenesis. IL-22-producing cells and Th22 cells were preferentially depleted from the gut mucosa during all stages of untreated HIV infection, and the increased production of IL-22 by CD3(-) mucosal immune cells (some of which were NKp44+) appeared to compensate for this loss during early HIV infection. However, during chronic infection all lymphocytic mucosal sources of IL-22 were depleted, gut epithelial integrity was compromised, and there was evidence of systemic microbial translocation. Finally, the protective and reparative role of IL-22 was confirmed in a well-defined in vitro enterocyte model, where exogenous IL-22 abrogated HIV-induced epithelial damage.

The mechanism(s) underlying the preferential loss of sigmoid IL-22-producing and Th22 cells in HIV-infected individuals was not determined in this translational study. Circulating Th22 cells expressed a highly HIV-susceptible phenotype, manifested by an increased expression of the HIV co-receptor CCR5 and HIV-binding integrin $\alpha 4 \beta 7$, and past studies have demonstrated that these markers are a good predictor of mucosal cell death and HIV gp-120 binding. ${ }^{20,34,35}$ However, expression of these surface markers only offers a partial explanation of IL-22 production loss, as $\mathrm{CD} 4(-)$ T cells and $\mathrm{CD} 3(-)$ non-T cells that would not be directly infected by HIV also demonstrated a reduced capacity to produce IL-22. Furthermore, we were not able to sort these functionally defined cells to examine provirus levels. Other possible mechanisms of reduced mucosal IL-22 production could include an inability of mucosal dendritic cell subsets to differentiate IL-22-producing cells, ${ }^{36}$ or a local 
a

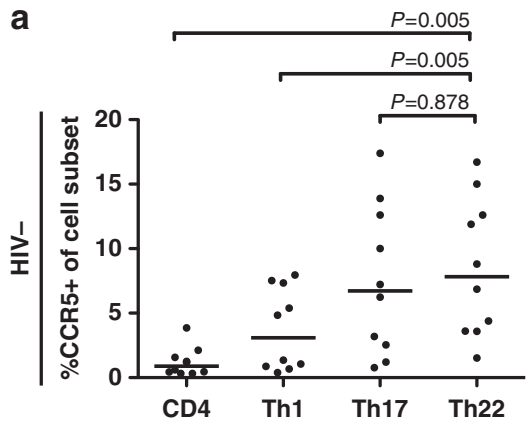

C

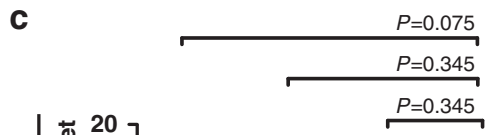

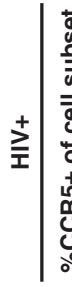

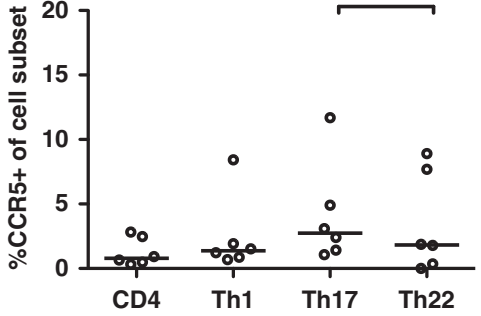

e

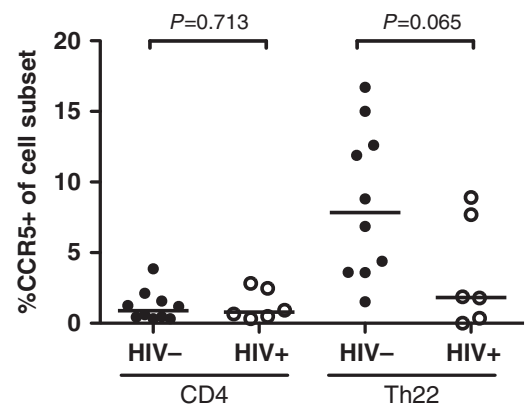

b

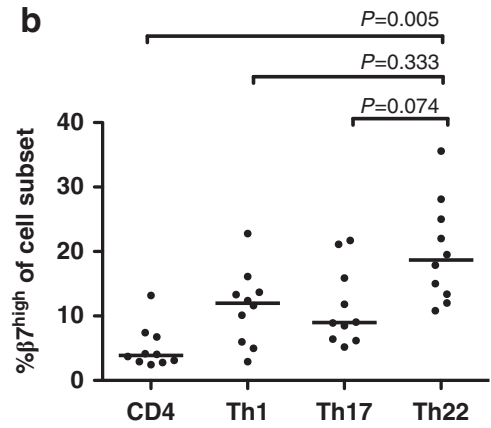

d
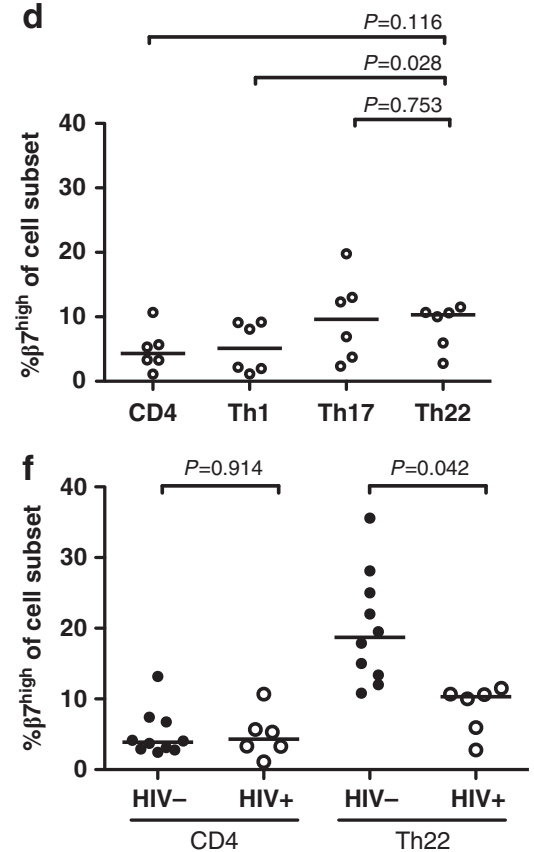

Figure 5 A higher frequency of circulating Th22 cells expressed the HIV co-receptor (CCR5) and binding molecule ( $\alpha 4 \beta 7)$ than other CD4 subsets in HIV-uninfected participants, but this phenotype was lost in HIV infection (Supplementary Figure 4 online for gating strategy). Frequency of (a and $\mathbf{c}$ ) CCR5 + and (b and d) $\beta 7^{\text {high }}+$ CD4 subsets of HIV-uninfected and HIV-infected individuals. Expression of (e) CCR5 and (f) $\beta 7^{\text {high }}$ on Th22 cells were decreased in HIV-infected individuals compared with uninfected individuals while they were comparable in CD4 cells.

deficiency in other immune signals necessary for IL-22 production. In the skin where IL-22 production has been studied in greater detail, Langerhans cells were able to differentiate Th22-polarized cells. ${ }^{37}$ Therefore, future studies of mucosal dendritic cell subsets and IL-22 production during HIV infection may be of value.

Th22 cells are not the only source of IL-22, and in our study other important sources of IL-22 in the gut mucosa included the Th1 and Th17 CD4+ subsets, as well as NK22 cells. ${ }^{21,38}$ Non-T-cell lineage-negative innate lymphoid cells, such as NKp44 + /CD56 + cells and lymphoid tissue-inducer-like cells, are well-described and important mucosal sources of IL-22. ${ }^{21,27}$ We were unable to define whether IL-22-producing cells or Th22 cells have an equivalent role in maintaining the gut barrier. However, the protective effect of recombinant IL-22 in our in vitro epithelial damage model and in other mucosal studies suggest that this cytokine has an important in vivo role regardless of the cell source..$^{22-24}$
While we did observe depletion of Th17 numbers in the sigmoid mucosa of chronically HIV-infected participants, the degree of depletion was lower than expected and did not extend to a reduced Th17 proportion, as has been previously observed. ${ }^{10,18,39}$ The reason for this is not known. One possibility relates to unmeasured differences in participants themselves. For example, recent studies in rhesus macaques demonstrated substantial variability in Th17 frequencies between animals ( $>5$-fold), and that the size of Th17 cell compartment before SIV infection was an important predictor of SIV clinical outcome. ${ }^{40}$ Whether such preexisting differences might explain variation in mucosal Th17 populations in humans is unknown. Notwithstanding the lower-than-expected Th17 depletion that we observed, the association of HIV infection with IL-22 depletion and reduced Th22 proportions and numbers in this cohort was clear and unambiguous.

A major consequence of HIV-induced gut epithelial damage is an influx of microbial products into the submucosa and 

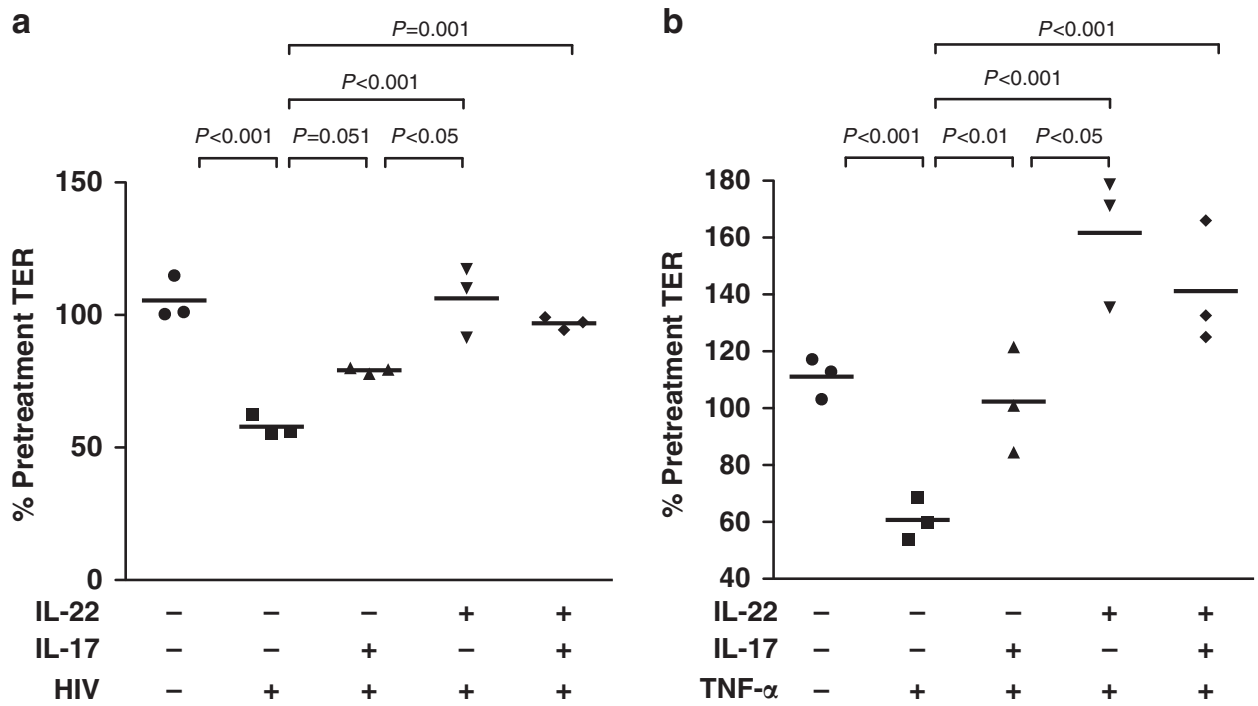

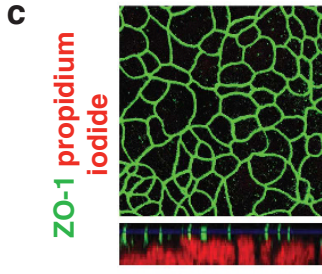

Mock

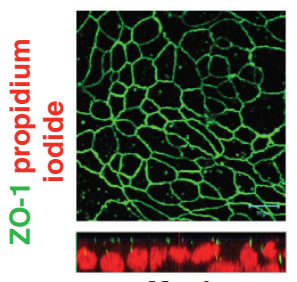

Mock

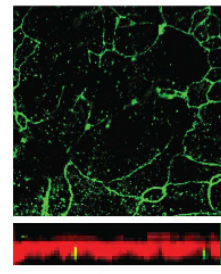

+HIV

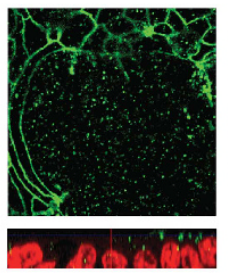

+ TNF- $\alpha$

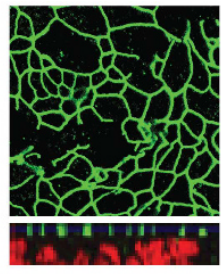

IL-17

+HIV

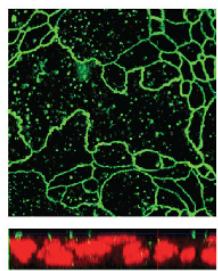

IL-17

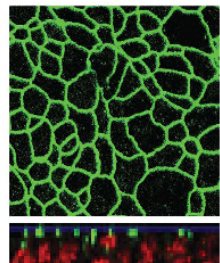

IL-22

+HIV

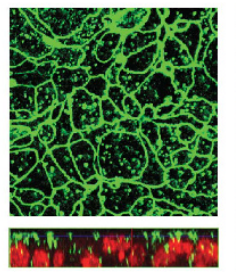

IL-22

+ TNF- $\alpha$

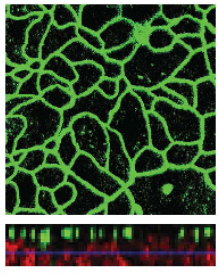

IL-17 and IL-22 $+\mathrm{HIV}$

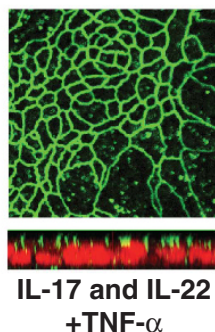

Figure 6 T84 human intestinal monolayers were pretreated with IL-17a and/or IL-22 and HIV, or cocultured with tumor necrosis factor- $\alpha$ (TNF- $\alpha$ ) for $24 \mathrm{~h}$, and transepithelial resistance (TER) measured. (a and b) IL-22 prevented TER loss more than IL-17a alone; however, both IL-17a and IL-22 prevented TER loss in HIV and TNF- $\alpha$-induced epithelial damage. (c) Immunofluorescence staining of the cells with anti-ZO-1 (green) and nuclear dye propidium iodide (red) demonstrates the loss of tight junction in the HIV or TNF- $\alpha$ control condition and a robust presence of ZO-1 in the cytokinetreated conditions.

subsequently into the blood, and circulating LPS may have an important role in driving HIV disease progression by increasing systemic immune activation. ${ }^{13,17}$ Previous studies have suggested that endotoxemia requires both the presence of structural barrier damage and mucosal immunodeficiency. ${ }^{16,17}$ In keeping with this, plasma LPS levels were only elevated in our chronic HIVinfection group, where mucosal IL-22 depletion and epithelial structural compromise were present simultaneously. In contrast, epithelial integrity was maintained during early HIV infection (despite mucosal immune compromise) and plasma LPS concentrations were normal. These data suggest that both mucosal structural damage and mucosal immune compromise must both be present to result in significant microbial translocation. The lack of an association between mucosal IL-22 production and plasma LPS levels in our study is likely to reflect the presence of multiple intermediate steps between microbial translocation and actual endotoxemia, including microbe-responsive Th17 cells, neutrophils, and phagocytic macrophages/monocytes in the gut mucosa, liver, and blood. ${ }^{6,16,17}$

In conclusion, our results suggest that IL-22 production and Th22 cells within the gut mucosa have an important role in the maintenance of mucosal epithelial integrity. Their preferential depletion during HIV infection may be important in mucosal immunopathogenesis, and suggests a possible avenue for novel HIV therapeutics.

\section{METHODS}

Participants. Study participants were recruited through the Maple Leaf Clinic (Toronto, Canada) and provided written informed consent. The Research Ethics Boards at St Michael's Hospital, Toronto, Canada and 
the University of Toronto approved the study protocol. Participants were categorized into the following groups: HIV-uninfected controls (HIV - , $n=8$ ); ART-naive HIV-infected participants (HIV,$+ n=24)$ in early HIV infection ( $<7$ months of HIV infection; $N=12$ ), and chronic HIV infection ( $>1$ year of HIV infection; $N=12$ ); long-term-treated individuals with an undetectable blood VL $(<50$ copies per $\mathrm{ml})$ for at least 6 years on ART (HIV + ART, $n=16)$.

Cell isolation from blood and sigmoid colon. Peripheral blood was collected into Acid Citrate Dextran solution A (BD Biosciences, La Jolla, $\mathrm{CA}$ ) and peripheral blood mononuclear cells isolated by Ficoll-Hypaque density centrifugation. ${ }^{10}$ Sigmoid biopsies were sampled $\sim 25-30 \mathrm{~cm}$ from the anal verge as previously described, ${ }^{10}$ weighed, and immediately placed into RPMI solution (RPMI-1640 media containing $100 \mathrm{U} \mathrm{ml}^{-1}$ penicillin, $100 \mu \mathrm{g} \mathrm{ml}^{-1}$ streptomycin, and $1 \times$ GlutaMAX-1; Invitrogen, Carlsbad, CA). Sigmoid mucosal mononuclear cells were isolated by two sequential Collagenase type II digestions at 0.5 and $1.0 \mathrm{mg} \mathrm{ml}^{-1}$ (Clostridiopeptidase A; Sigma-Aldrich, St Louis, MI) for $30 \mathrm{~min}$ each at $37^{\circ} \mathrm{C}$ on a shaking heated block. Mucosal cells were passed through a $100-\mu \mathrm{m}$ filter and enumerated and the median cell yield was about 46.2 million cells per $g$ tissue and was similar across study groups.

Flow cytometry immune studies. Isolated blood and sigmoid mononuclear cells were stimulated with phorbol 12-myristate 13 -acetate $\left(1 \mathrm{ng} \mathrm{ml}^{-1}\right)$ and ionomycin $\left(1 \mu \mathrm{M} \mathrm{ml}^{-1}\right.$; Sigma-Aldrich), or negative control media alone for $6 \mathrm{~h}$ at $37^{\circ} \mathrm{C}$ and $5 \% \mathrm{CO}_{2}$, with the last $5 \mathrm{~h}$ including Brefeldin A $\left(1 \mu \mathrm{m} \mathrm{ml}^{-1}\right)$. All stimulations occurred in RPMI solution supplemented with 10\% fetal bovine serum (FBS). Cells were washed with ice-cold $1 \%$ FBS- $1 \times$ phosphate-buffered saline (PBS), permeabilized, stained for $30 \mathrm{~min}$ with fluorochrome-labeled monoclonal antibodies and a viability dye (Invitrogen), and fixed in $2 \%$ paraformaldehyde. Antibodies included CD3, CD4, CD8, HLADR, CCR5, IL-17a, IL-22, IFN- $\gamma$, CD49d, B7, and TNF- $\alpha$ (See Supplementary Table 2 online; BD BioSciences; E-Bioscience, San Diego, CA; and Beckman Coulter, Fullerton, CA). Cells were acquired on a FACSCanto II or LSR II (BD Biosciences). Data analysis was performed using Flow Jo analytical software v9.0.2 (TreeStar, Ashland, OR), and flow cytometric gating was determined using media and/or all fluorescence minus one controls, and an event count below 15 was not further sub-analyzed. Values for functional cell subsets were background-corrected.

Calculation of absolute number of sigmoid cell subsets. The absolute number of gut cells were determined by multiplying the percentage of live lymphocytes determined by flow cytometry to the number of gut cells per gram of tissue, as previously described. ${ }^{41}$ Biopsies were pre-weighed before tissue digestion and enumerated, and the total gut cell count per $g$ tissue was determined by dividing the number of counted sigmoid cells to the total tissue weight. This value was then multiplied by the percentage of cells in the live lymphocyte gate and multiplied by subsequent gates based on flow cytometric analysis.

Immunofluorescence. Fresh sigmoid biopsies were embedded in Tissue-Tek OCT compound and flash frozen in an ice-cold bath of 2methylbutane. Biopsies were sectioned $(5 \mu \mathrm{m})$ using Leica $1035 \mathrm{~S}$ cryostat (Leica Microsystems, Exton, PA), fixed in acetone, and air-dried. Sections were rehydrated in PBS, blocked with PBS-T (PBS with $0.05 \%$ Tween-20; Sigma) supplemented with $4 \%$ BSA, 5\% normal goat serum, and $10 \%$ normal human plasma for $30 \mathrm{~min}$. Tight junction antibodies included anti-ZO-1 and anti-claudin-2 rabbit polyclonal antibodies (Zymed, San Francisco, CA), and mouse monoclonal cytokeratin PE (Cedarlane, Ontario, CA), and T-cell antibodies included anti-CD3 biotin (Biolegend, San Diego, CA) and anti-CCR10 APC (Biolegend). Secondary antibodies included streptavidin FITC or goat anti-rabbit IgG AF488. Finally, slides were stained with 46-diamidino-2-phenylindole (Invitrogen) and mounted with Acqueous Mounting Medium (R\&D Systems, Minneapolis, MN). Images of stained sections were obtained by the use of a Leica DM-R digital fluorescence microscope with Retiga EXi digital camera (QImaging, Surrey, BC) using OpenLab (Improvision, Lexington, MA). Images were processed using Adobe Photoshop CS5 (image crop/merge layers), ensuring that all panels were processed in the same manner.

In vitroT84 monolayer cytokine study. Human colon-derived crypt-like T84 epithelial monolayers were cultured as previously described ${ }^{15}$ in a 1:1 (vol/vol) mixture of 10\% FBS supplemented DMEM and Ham's F-12 medium at $37^{\circ} \mathrm{C}$ in $5 \% \mathrm{CO}_{2}$ for $5-6$ days until confluency, pretreated with $10 \mathrm{ng} \mathrm{ml}^{-1}$ of recombinant human IL-17a, IL-22, both cytokines combined (R\&D Systems), or with media alone, and then exposed to HIV strain ADA ( $\mathrm{R} 5$ tropic; $\mathrm{MOI}=1 ; 10 \mathrm{ng} \mathrm{ml}^{-1}$ ). In separate experiments, IL-22 and IL-17a were added individually or in combination with recombinant TNF- $\alpha$ apically and left on monolayers through the experiment. Controls included cultures that were mock-treated with medium without HIV or TNF- $\alpha$ and positive controls included HIV or TNF- $\alpha$ alone; control conditions were devoid of cytokine. TER was measured using a volt ohm meter (EVOM; World Precision Instruments, Sarasota, FL) before exposure, 24 and 48 h, post-exposure to HIV or TNF- $\alpha$, and compared with mock infection. Studies were completed in three separate experiments and each experiment contained duplicate cultures for each condition. Paraformaldehyde (4\%)-fixed monolayers were stained with rabbit anti-human ZO-1 (Zymed) in blocking solution for $1 \mathrm{~h}$ at room temperature, followed by AF488 goat anti-rabbit IgG (Molecular Probes, Eugene, OR). All samples were imaged on an inverted confocal laserscanning microscope (LSM 510) using standard operating conditions as previously reported. ${ }^{15}$

Microbial translocation markers. Assays were performed according to manufacturers' instructions, in duplicate. Plasma samples were diluted $5 \times$ in endotoxin-free water, heat inactivated at $65^{\circ} \mathrm{C}$ for $15 \mathrm{~min}$, and assayed to quantify LPS levels using the limulus amebocyte lysate assay kit (Cambrex, Charles City, IO). Commercially available ELISA kit was used to measure levels of sCD14 (R\&D Systems).

Statistical analysis. Spearman's rank-correlation, Wilcoxon, and MannWhitney $U$-tests were performed for all human studies and the one-way analysis of variance with the Bonferroni post-hoc test was applied for the T84 in vitro study using IBM SPSS Statistics 18.0 for Mac (SPSS, Chicago, IL). $P<0.05$ was considered significant.

SUPPLEMENTARY MATERIAL is linked to the online version of the paper at http://www.nature.com/mi

\section{ACKNOWLEDGMENTS}

We thank Dr David O Willer and Dr Lyle McKinnon for their assistance in proof reading this manuscript, and Dr Prameet Sheth and Dr Ali Sakhdari for their advice. We acknowledge all individuals who participated in this study. This work was supported in part by the Ontario HIV Treatment Network (RK, ROGB-G123); the Canadian Institutes of Health Research/ Canadian Digestive Health Foundation (CIHR/CDHF; CJK, salary award); the Ontario Graduate Student Science and Technology Scholarships (CJK, salary award); a CIHR (Emerging HIV Team Grant \#HET85518 and \#67157); and the Canadian Research Chair Program (RK, salary support). The funders had no role in the study design, data collection and analysis, decision to publish, or preparation of the manuscript.

\section{DISCLOSURE}

The authors declared no conflict of interest.

2012 Society for Mucosal Immunology

\section{REFERENCES}

1. Mehandru, S. et al. Primary HIV-1 infection is associated with preferential depletion of CD4+ T lymphocytes from effector sites in the gastrointestinal tract. J. Exp. Med. 200, 761-770 (2004). 
2. Sankaran, S. et al. Rapid onset of intestinal epithelial barrier dysfunction in primary human immunodeficiency virus infection is driven by an imbalance between immune response and mucosal repair and regeneration. J. Virol. 82, 538-545 (2008).

3. Veazey, R.S. \& Lackner, A.A. The gastrointestinal tract and the pathogenesis of AIDS. AIDS 12 (Suppl A), S35-S42 (1998).

4. Veazey, R.S. et al. Gastrointestinal tract as a major site of CD4+ T cell depletion and viral replication in SIV infection. Science 280, 427-431 (1998).

5. Epple, H.J. et al. Impairment of the intestinal barrier is evident in untreated but absent in suppressively treated HIV-infected patients. Gut 58, 220-227 (2009).

6. Ancuta, P. et al. Microbial translocation is associated with increased monocyte activation and dementia in AIDS patients. PLoS One 3 , e2516 (2008).

7. Ross, A.C. et al. Endothelial activation markers are linked to HIV status and are independent of antiretroviral therapy and lipoatrophy. J. Acquir. Immune. Defic. Syndr. 49, 499-506 (2008).

8. Ross, A.C. et al. Relationship between inflammatory markers, endothelia activation markers, and carotid intima-media thickness in HIV-infected patients receiving antiretroviral therapy. Clin. Infect. Dis. 49, 1119-1127 (2009).

9. Wallet, M.A. et al. Microbial translocation induces persistent macrophage activation unrelated to HIV-1 levels or T-cell activation following therapy. AIDS 24, 1281-1290 (2010)

10. Chege, D. et al. Sigmoid Th17 populations, the HIV latent reservoir, and microbial translocation in men on long-term antiretroviral therapy. AIDS 25, 741-749 (2011).

11. Gordon, S.N. et al. Severe depletion of mucosal CD4+ T cells in AIDS-free simian immunodeficiency virus-infected sooty mangabeys. J. Immunol. 179, 3026-3034 (2007).

12. Pandrea, I.V. et al. Acute loss of intestinal CD4+ T cells is not predictive of simian immunodeficiency virus virulence. J. Immunol. 179, 3035-3046 (2007).

13. Brenchley, J.M. et al. Microbial translocation is a cause of systemic immune activation in chronic HIV infection. Nat. Med. 12, 1365-1371 (2006).

14. Epple, H.J. et al. Acute HIV infection induces mucosal infiltration with CD4+ and CD8+ T cells, epithelial apoptosis, and a mucosal barrier defect. Gastroenterology 139, 1289-1300 (2010)

15. Nazli, A. et al. Exposure to HIV-1 directly impairs mucosal epithelial barrier integrity allowing microbial translocation. PLoS Pathog. 6, e1000852 (2010).

16. Hofer, U. et al. Inadequate clearance of translocated bacterial products in HIV-infected humanized mice. PLoS Pathog. 6, e1000867 (2010).

17. Estes, J.D. et al. Damaged intestinal epithelial integrity linked to microbial translocation in pathogenic simian immunodeficiency virus infections. PLoS Pathog. 6, e1001052 (2010)

18. Brenchley, J.M. et al. Differential Th17 CD4 T-cell depletion in pathogenic and nonpathogenic lentiviral infections. Blood 112, 2826-2835 (2008).

19. Ye, P. et al. Interleukin-17 and lung host defense against Klebsiella pneumoniae infection. Am. J. Respir. Cell Mol. Biol. 25, 335-340 (2001).

20. Kader, M. et al. Alpha4(+)beta7(hi)CD4(+) memory T cells harbor most Th-17 cells and are preferentially infected during acute SIV infection. Mucosal Immunol. 2, 439-449 (2009).

21. Sonnenberg, G.F., Fouser, L.A. \& Artis, D. Border patrol: regulation of immunity, inflammation and tissue homeostasis at barrier surfaces by IL-22. Nat. Immunol. 12, 383-390 (2011).
22. Pickert, G. et al. STAT3 links IL-22 signaling in intestinal epithelial cells to mucosal wound healing. J. Exp. Med. 206, 1465-1472 (2009).

23. Sugimoto, K. et al. IL-22 ameliorates intestinal inflammation in a mouse model of ulcerative colitis. J. Clin. Invest. 118, 534-544 (2008).

24. Wolk, K., Kunz, S., Witte, E., Friedrich, M., Asadullah, K. \& Sabat, R. IL-22 increases the innate immunity of tissues. Immunity $\mathbf{2 1 , 2 4 1 - 2 5 4}$ (2004).

25. De Luca, A. et al. IL-22 defines a novel immune pathway of antifungal resistance. Mucosal Immunol. 3, 361-373 (2010).

26. Ota, N. et al. IL-22 bridges the lymphotoxin pathway with the maintenance of colonic lymphoid structures during infection with Citrobacter rodentium. Nat. Immunol. 12, 941-948 (2011).

27. Trifari, S., Kaplan, C.D., Tran, E.H., Crellin, N.K. \& Spits, H. Identification of a human helper $\mathrm{T}$ cell population that has abundant production of interleukin 22 and is distinct from $\mathrm{T}(\mathrm{H})-17, \mathrm{~T}(\mathrm{H}) 1$ and $\mathrm{T}(\mathrm{H}) 2$ cells. Nat. Immunol. 10, 864-871 (2009).

28. Eyerich, S. et al. Th22 cells represent a distinct human T cell subset involved in epidermal immunity and remodeling. J. Clin. Invest. 119, 3573-3585 (2009).

29. Duhen, T., Geiger, R., Jarrossay, D., Lanzavecchia, A. \& Sallusto, F. Production of interleukin 22 but not interleukin 17 by a subset of human skin-homing memory T cells. Nat. Immunol. 10, 857-863 (2009).

30. de Jong, A., Pena-Cruz, V., Cheng, T.Y., Clark, R.A., Van Rhijn, I. \& Moody, D.B. CD1a-autoreactive T cells are a normal component of the human alphabeta T cell repertoire. Nat. Immunol. 11, 1102-1109 (2010).

31. Guillot-Delost, M. et al. Human CD90 identifies Th17/Tc17 T cell subsets that are depleted in HIV-infected patients. J. Immunol. 188, 981-991 (2012).

32. Bengsch, B., Seigel, B., Flecken, T., Wolanski, J., Blum, H.E. \& Thimme, R. Human Th17 cells express high levels of enzymatically active dipeptidylpeptidase IV (CD26). J. Immunol. 188, 5438-5447 (2012).

33. Sandler, N.G. et al. Plasma levels of soluble CD14 independently predict mortality in HIV infection. J. Infect. Dis. 203, 780-790 (2011).

34. Monteiro, P. et al. Memory CCR6+CD4+ T cells are preferential targets for productive HIV type 1 infection regardless of their expression of integrin \{beta\}7. J. Immunol. 186, 4618-4630 (2011)

35. McKinnon, L.R. et al. Characterization of a human cervical CD4+ T cell subset coexpressing multiple markers of HIV susceptibility. J. Immunol. 187, 6032-6042 (2011).

36. Klatt, N.R. et al. Loss of mucosal CD103+ DCs and IL-17+ and IL-22+ lymphocytes is associated with mucosal damage in SIV infection. Mucosal Immunol. advance online publication, 30 May 2012; doi:10/1038/mi.2012.38 (2012).

37. Fujita, H., Nograles, K.E., Kikuchi, T., Gonzalez, J., Carucci, J.A. \& Krueger, J.G. Human Langerhans cells induce distinct IL-22-producing CD4+ T cells lacking IL-17 production. Proc. Natl Acad. Sci. USA 106, 21795-21800 (2009)

38. Cella, M. et al. A human natural killer cell subset provides an innate source of IL-22 for mucosal immunity. Nature 457, 722-725 (2009).

39. Favre, D. et al. Tryptophan catabolism by indoleamine 2,3-dioxygenase 1 alters the balance of $\mathrm{TH} 17$ to regulatory T cells in HIV disease. Sci. Transl. Med. 2, 32 ra36 (2010)

40. Hartigan-O'Connor, D.J., Abel, K., Van Rompay, K.K., Kanwar, B. \& McCune, J.M. SIV replication in the infected rhesus macaque is limited by the size of the preexisting TH17 cell compartment. Sci. Transl. Med. 4, $136 r a 169$ (2012).

41. Ciccone, E.J. et al. Cycling of gut mucosal CD4+ T cells decreases after prolonged anti-retroviral therapy and is associated with plasma LPS levels. Mucosal. Immunol. 3, 172-181 (2010). 